\title{
Effect of Neighborhood on In-Network Processing in Sensor Networks
}

\author{
Muhammad Jafar Sadeq and Matt Duckham \\ Department of Geomatics, The University of Melbourne, Victoria, 3010, Australia \\ m.sadeq@pgrad.unimelb.edu.au, matt@duckham.org
}

\begin{abstract}
Wireless sensor networks are growing from a few hand-placed devices to more large-scale networks in terms of coverage and node density. For various concerns, such as scalability, larger network sizes require some management of the large volume of data that a sensor network delivers. One way to manage this data is processing information in the network. This paper investigates how a sensor network's network architecture (specifically, the neighborhood structure) can influence the conclusions that a sensor network makes from its measurements. The results demonstrate that non-planar structures are infeasible for routing and some in-network processing applications. Structures with low average edge lengths give better quantitative results, while those with high edge densities give better qualitative results.
\end{abstract}

\section{Introduction}

Wireless sensor networks (WSNs) are untethered networks of miniaturized sensorenabled computers. WSNs are increasingly being used for geospatial applications, such as environmental monitoring. WSNs today typically use a periodic "senseand-transmit" approach to transmit temporal snapshots of the entire network's readings to a centralized computing system [1][2]. The centralized computer system (such as a conventional GIS) is then tasked with analyzing the snapshots to determine what significant events have occurred. As such, many of today's WSN deployments can be regarded as sophisticated data loggers [3]. There are at least three important drawbacks to this approach:

- Energy resources: WSNs are highly resource constrained systems, in particular with respect to sensor node energy resources. Wireless communication is one of the most energy-intensive activities of a sensor node, so continually relaying data to a central system can dramatically shorten the useful lifetime of a WSN.

- Information overload: The fine-grained spatiotemporal detail becoming available from larger sensor networks means that individual data items become less and less meaningful. Transmitting all data can lead to high levels of redundancy and ultimately information overload.

- Sensor/actuator networks: The results of the analysis of sensor network data are often required by the network itself in order modify the behavior of 
the network (e.g., activate or deactivate sensors to adapt the granularity of monitoring depending on the activity of the environment, sometimes called georesponsive sensor networks [4]). Removing information from the network, processing it centrally, then returning it to the network is an inefficient drain on network resources.

A growing proportion of current research in WSNs is dedicated to addressing these problems using in-network processing, where sensor nodes themselves collaborate in a decentralized manner to perform partial or complete processing of sensor data. This paper investigates how the structure of the network (links between nodes) can influence the results of in-network processing of spatial information and events.

Due to issues such as scalability, in-network processing often involves distributed processing with nodes using information from their neighbors. The question then arises as to which nodes are considered neighbors of a particular node. Often, the simplest solution is taken, where a node has as its neighbors all nodes that it can communicate with directly [5][6]. However, for routing, neighborhood will sometimes need to be limited to planar graphs (as discussed further in Sect. 2). Therefore, multiple definitions of neighborhood may exist in the same WSN. This paper evaluates the use of different neighborhood structures for in-network processing. Many of these structures are already used in routing research, thus gaining multiple uses out of a single infrastructure.

The importance of neighborhood structures to spatial applications can be seen when we realize that, for most environmental variables (such as temperature or light intensity), nodes take only point readings (with exceptions such as cameras). There is often no knowledge of the readings between nodes, either in the WSN or in any central server. Neighborhood structures may be used to estimate these unsampled regions. Techniques such as Voronoi diagrams [7] can partition the field into coverage areas in order to interpolate readings for the unsensed areas. Techniques such as kriging [8] estimate values at unsampled points. Given sufficient point estimates, point readings can be interpolated to a field. These techniques, however, may be computationally expensive when a node needs to do frequent interpolations in response to a dynamic environment.

From a qualitative spatial reasoning perspective, a fundamental interpolation process concerns the presence or absence of a boundary between neighboring nodes. Consider a binary region, for example a hot region (temperature $>25^{\circ} \mathrm{C}$ ) and its complement (temperature $\leq 25^{\circ} \mathrm{C}$ ). If two nodes are connected topologically, and one senses that it is in the hot region and the other senses that it is out of the hot region, it is certain that there exists a boundary of the hot region boundary between them. If they both sense the hot region, it can be inferred that there is no boundary between them. An example is shown in Fig. 1. Based on a purely local, qualitative knowledge observations in their immediate neighborhood, Nodes $A$ and $D$ deduce that there must be a boundary between. Similarly, Nodes $A$ and $C$ can infer that there is no boundary between them. Thus, by answering a qualitative question ("Is there a boundary between myself 
and my neighbor?"), the network topology can be used to build a picture of a region.

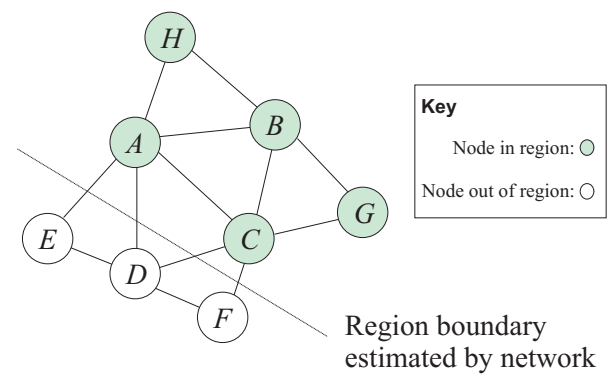

Fig. 1. Use of network edges to build a region

In order to evaluate various neighborhood structures, this paper quantitatively tests the ability of a WSN to approximate the boundary of a region when different structures are applied to it. Boundary was chosen as an evaluation criterion because it is a fundamental construct for the detection of spatial events in region evolution - events occur at a region's boundary or events create new boundaries. Boundary has salience for human spatial cognition and can be used to represent various spatial properties of the region such as its shape or its area. This work also tests the neighborhood structures' ability to qualitatively detect topological events occurring to a region.

It has been found that non-planar structures are infeasible not only for routing but can also cause inconsistencies in a WSN's knowledge and state. Neighborhood structures with a lower average edge length gives better quantitative results. Those with high edge densities give better qualitative results. Higher edge densities usually increase the average edge length, so high edge densities result in poorer quantitative performance.

\section{Background}

A lot of research effort has been given to developing neighborhood structures for WSNs because of their importance to routing. It is especially important to greedy routing, where a message is sent to a node geographically closer to the destination than the current node. An example of a greedy routing algorithm is Greedy Perimeter Stateless Routing (GPSR) [9].

Greedy forwarding can fail if the message reaches a "void" where no node is closer to the destination than the current node. GPSR therefore includes procedures to route around the edge of the void (Geographic Face Routing) [9][10]. Such forms of geographic routing (routing to a specific location) therefore require specific properties from the network graph of a WSN. One is that the number of voids must be minimized because face routing is more costly than 
greedy forwarding. Another is that the graph must be planar (there must be no crossing edges), otherwise face routing can fail. This in turn requires that there are no uni-directional links [10].

Researchers use network graphs to test their algorithms and protocols. Occasionally, researchers compare various neighborhood structures for the purposes of routing. However, to our knowledge, there is no research on the effect of the various neighborhood structures on the results of in-network processing. This may be because there is little research so far into in-network processing of higher level spatial information that rely on neighborhood structures.

This paper investigates how well different neighborhood structure approximate the boundary of a region and how well they detect topological events. A number of boundary detection algorithms have been proposed in the literature. In the classifier approach, nodes estimate the location of a straight line representing the local portion of the region boundary [5]. A node's distance from that line is used to determine whether it is a boundary node. In another approach, a node compares the average of the readings from its neighborhood to a threshold in order to determine whether it is on the boundary [6].

By contrast, this paper adopts a qualitative approach where boundary nodes are identified purely from their relationship to immediate neighbors (i.e., whether or not they neighbor a node on the opposite side of a boundary). In addition to simplifying the model and computation, adopting this qualitative approach can help improve the network's robustness to imperfection in sensor data and boundary indeterminary.

\section{$3 \quad$ Neighborhood Structures}

Modeling a network as a graph $G=(S, E)$, with a set of vertices $S \subset R^{2}$ representing sensor nodes as points in the plane and edges $E \subseteq S \times S$ representing the potential for direct one-hop communication between nodes, the following neighborhood structures are commonly used.

- Localized Delaunay triangulation: The Delaunay triangulation $D T(S)$ is the unique triangulation of $S$ such that no point in $S$ is inside the circumcircle of any triangle in $D T(S)$ [11]. A localized Delaunay triangulation (LDT) is a graph resulting from attempting to build a Delaunay triangulation (DT) using limited information (information local to a node). The LDT does not have a complete convex hull since some edges that exceed the communication range of the nodes are not included in the graph. Depending on the algorithm, it may not even be a triangulation.

- Gabriel graph: $E$ includes edges $(x, y)$ and $(y, x)$ between two points $x \in S$ and $y \in S$ if the circle with $x y$ as the diameter is free of other points in $S$ [12]. $G G(S)$ is a subgraph of $D T(S)$.

- Relative neighborhood graph: $E$ includes edges $(x, y)$ and $(y, x)$ if the lune (the intersection of the two circles with centers $x$ and $y$ and with $x y$ as radius) is free of other points in $S . R N G(S)$ is a subgraph of $G G(S)$ [12]. 
- Localized greedy triangulation: The greedy triangulation $G T(S)$ of a set of points $S$ is built by first creating a list of all edges between all nodes in the network. The shortest edge is removed from the list and it is added to the triangulation if it does not cross an edge that has already been inserted into the triangulation. This continues until all the edges have been exhausted or the number of edges in the triangulation is $3 \times N-6$, where $N=|S|$. Thus the greedy triangulation (GT) attempts to minimize total edge length in a greedy manner [11]. The localized GT (LGT), built by nodes using local information, is not a complete triangulation, similar to LDT.

- Unit disk graph: UDG is a non-planar graph where there is an edge between two points as long as the points are at most a unit distance apart. In a WSN, this is a graph where each node has edges to all nodes that it can communicate with.

The graphs can be compared in Fig. 2, which shows graphs built in a distributed manner by a WSN simulation.

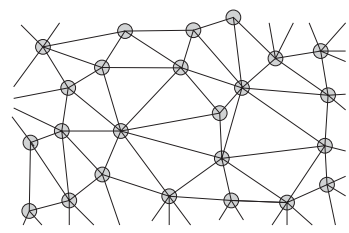

(a) DT

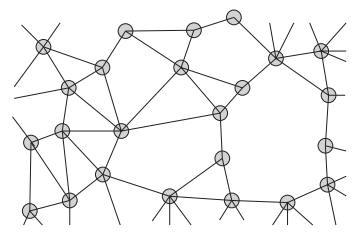

(b) GG

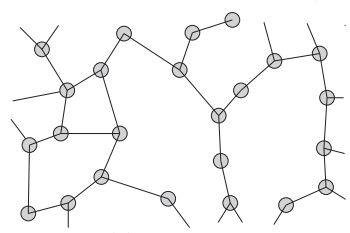

(c) RNG

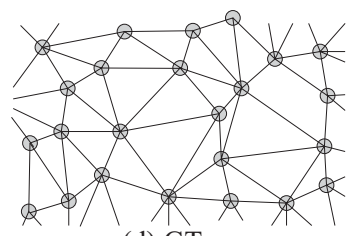

(d) GT

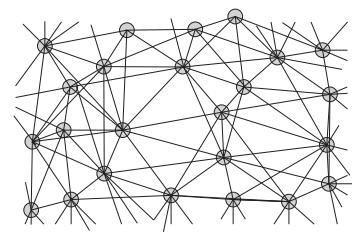

(e) UDG

Fig. 2. Neighborhood structures built in a distributed manner

These five cover a variety of neighborhood structures. DT is well-known in spatial applications, so it is sensible to test a distributed version in a WSN. GG and RNG are built using completely local criteria and therefore lend themselves to implementation in WSNs using distributed processing. GT's usage of edge length as a criterion may make it suitable for WSNs because many localization algorithms determine distance (using, for example, received signal strength) before deriving coordinate information [13]. Finally, UDG is the most basic case where all possible edges between nodes exist. 


\section{Methodology}

The neighborhood structures were compared against each other both quantitatively and qualitatively. The quantitative properties of the neighborhood structures were evaluated using a WSN's approximation of the boundary of a region. Their qualitative properties were tested by determining their ability to detect certain topological events. These tests were done by simulation.

\subsection{Simulation Environment}

It was infeasible to perform the tests using real sensor nodes due to the requirement for a large number of nodes to make the spatial properties of the neighborhood structures meaningful. Therefore, the tests were done by simulation. Repast (http://repast.sourceforge.net/), a Java-based agent modeling tool, was used. Each sensor node in the simulation was made an independent Java object. The field being simulated was also independent from the sensor nodes. The nodes interact among each other and with the environment in a decentralized manner.

\subsection{Decentralized Computation of Neighborhood Structures}

In our simulations, the neighborhood structures were built by the nodes themselves in a decentralized manner. Researchers have developed many decentralized algorithms to build network graphs that meet the routing requirements that have been mentioned in Sect. 2. The Cross-link Detection Protocol (CLDP) can generate a planar subgraph of any arbitrary graph of the network [10]. A localized DT built by a WSN can be ensured to be planar as long as a node uses information from nodes that are two communication hops away [14]. The Gabriel graph (GG) has also been used to develop network architectures [15], as it does not need a special algorithm to be built by sensor nodes locally.

In our simulations, the GG and RNG were built by simply by each node applying the GG and RNG rules (Sect. 3) to all nodes that it can communicate with directly. Each node $x \in S$ starts with a list of these nodes, which it can acquire in an initial handshaking phase. $x$ tests each node in this list to check whether an edge between them is permitted in GG or RNG.

For the LDT, for every pair of nodes $y$ and $z$ in $x$ 's communication range, if there were no nodes in the circumcircle of $\triangle x y z$, edges $(x, y)$ and $(x, z)$ were added to $E$. Thus, for GG, RNG and LDT, the node does not compute the complete graph of all the points in its communication range.

If $x$ uses nodes in its communication range to compute the LDT, a nonplanar graph may result. This happens when $x$ rejects a triangle $\triangle x y z$ due to a node $w$ in $\triangle x y z$ 's circumcircle, but $y$ or $z$ accepts $\triangle x y z$ because $w$ is out of its communication range. This situation results in unidirectional links. If nodes that are two communication hops away are included in the triangulation procedure, planarization is ensured [14]). For simplicity, however, in our simulations $x$ removed all unidirectional edges (each edge $(x, y)$ for which $y$ did not have an edge $(y, x))$. Additionally, triangles whose circumcircles include more than 
three nodes were not included in the graph because they can result in crossing links. These procedures gave us a planar graph, but not a complete triangulation. Rather, the LDT is a graph based on the rules for Delaunay triangulation.

LGT requires the nodes to compute all possible edges between all its neighbors and create the complete graph to ensure no edges cross. Although fast algorithms for GT exist in the literature, our objective in this work was not to use the most efficient algorithms but rather to test the neighborhood structures. Therefore, we used algorithms that were the simplest for us to implement, i.e., we applied the basic rules of the neighborhood structures.

Finally, UDG was built by each node creating an edge to each other node within .

\subsection{Quantitative Test: $L^{2}$ Error Norm}

To quantitatively determine the accuracy of the region boundaries as approximated by the WSN under various neighborhood structures, the shape, location and size of the approximated region boundary were compared to the actual boundary. The $L^{2}$ error norm provided a simple means to test all of these properties. The $L^{2}$ error norm is the area of the region enclosed between the boundaries of two shapes. Given a region that a WSN is monitoring, it determines an approximation $P$ of the original region $O$. The $L^{2}$ error norm was computed in

this paper by finding the area of the symmetric difference between $O$ and $P$ as a proportion of the total area of $O$, i.e.,

$$
L^{2} \text { error norm }=\frac{\operatorname{area}((O-P) \cup(P-O))}{\operatorname{area}(O)} .
$$

An $L^{2}$ error norm of zero means that not only are the areas of the two shapes equal, but also that their boundaries are in complete agreement.

\subsection{Qualitative Test: Detecting Topological Events}

There exist six fundamental topological events for dynamic areal objects: appearance and disappearance; merge and split; and self-merge and partial split [16].

1. Appearance/disappearance: A region or hole appears or disappears.

2. Merge/split: Two disconnected region components meet and become connected, or a region component splits into two disconnected components.

3. Self-merge/partial-split: A hole is formed in a region by engulfing part of it's exterior (self-merge) or a hole is destroyed in a region by its merging with the region's exterior (partial-split).

Examples of the six fundamental topological events are shown in Fig. 3.

In a concurrent work, we have developed an algorithm to detect these events and distinguish them from each other and from non-topological events (expansion and contraction). For several reasons, including space limitation, only merge 
a.

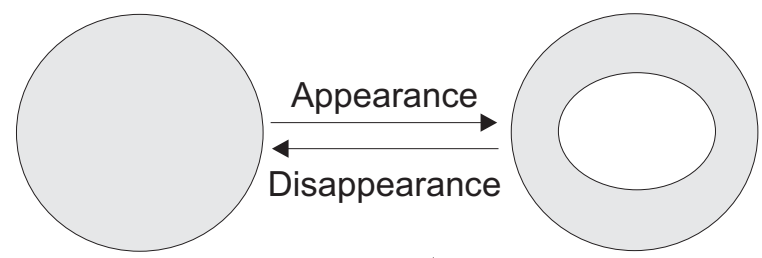

b.

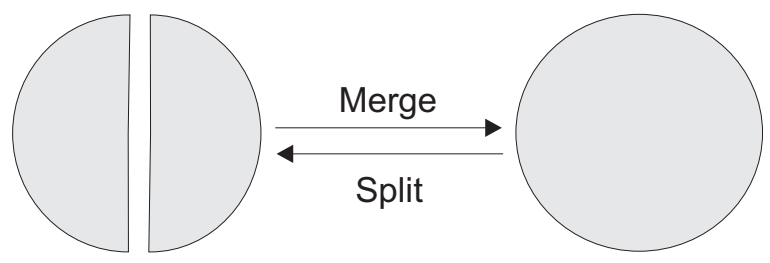

c.

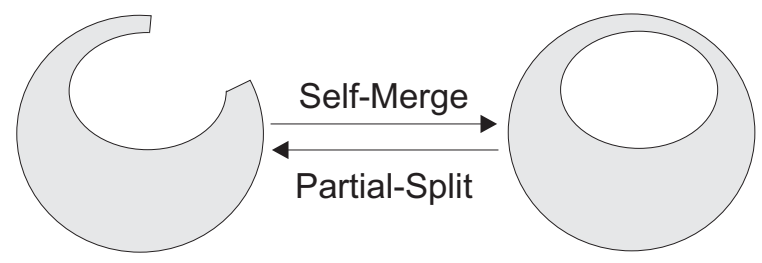

Fig. 3. Six fundamental topological events for dynamic areal objects

and split are investigated in this paper. Partial-split is not investigated because the algorithm to detect it is not independent, relying on previous events being identified correctly. Self-merge is left out because it is similar to split in its requirements from a neighborhood structure. Misidentified appearances and disappearances usually result in incorrect detections of other events (split and merge), therefore it was felt that merge and split were suitably representative of topological events to be used to analyze the neighborhood structures' effectiveness.

The complete algorithm to detect and distinguish spatial events is beyond the scope of these paper. In fact, the algorithm for detecting a merge is not needed to understand why a merge can be incorrectly detected, so that is left out too. Only a condensed version for the detection of split is presented.

Split Detection The algorithm for detection of a split is complicated due to the difficulty in distinguishing it from contraction. In both Fig. 4(a) and Fig. 4(b), Node $A$ has detected a change in the environment. Consequently, the region being monitored no longer includes $A$. To differentiate split and contraction, Node $A$ maintains its neighbors in a list sorted by direction (called the cyclic ordering of the neighbors). With this sorted list, it determines how many blocks of region/hole surround it. 
(a)

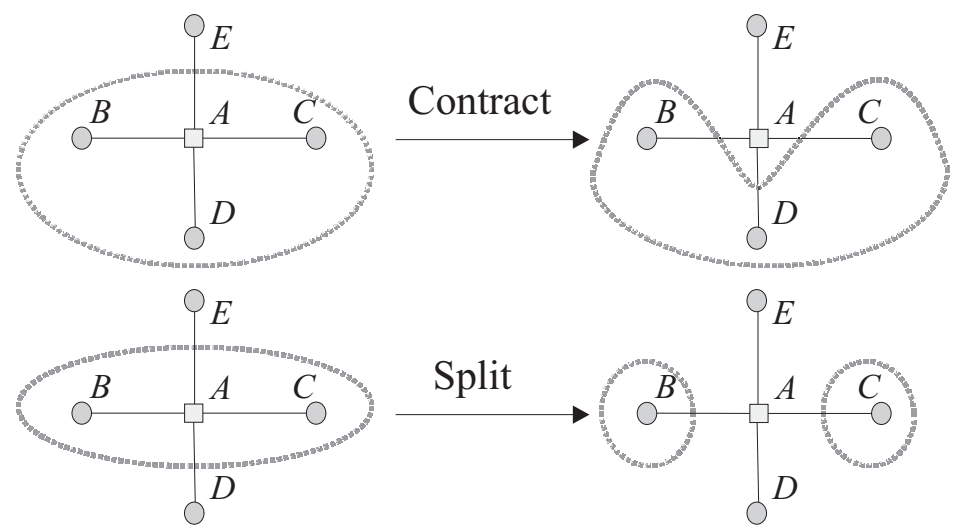

Fig. 4. Split/contract

If there are two such blocks, the region has contracted - in Fig. 4(a), $A$ is surrounded by an in-region block consisting of $B, D$ and $C$ and an out-of-region block consisting of only $E$. If there are four or more blocks (Fig. 4(b): $B, D, C$ and $E$ are all in separate blocks around $A$ ), the region has split.

To demonstrate why four blocks are necessary, consider $D$. If $D$ were not in $A$ 's neighborhood, $A$ would not be able to determine whether a split has occurred. This is because, from $A$ 's point of view, there is a consecutive block of region around it consisting of $B$ and $C$. It therefore assumes that a contraction has occurred. Additionally, the absence of $D$ means that $A$ cannot locally determine whether the region is connected beyond its immediate neighborhood.

Further cases (such as if $D$ is actually in a hole in the region) are easily resolved, but those issues are beyond the scope of the paper. In our simulations to evaluate neighborhood structures, we controlled the region evolution so that only merge and split occurred. Therefore, in this paper, it is not necessary to discuss the complete algorithm. Instead, it is sufficient to realize that, to detect a split, a node must have at least four neighbors.

\section{Boundary Approximation and Analysis}

One hundred random deployments of 500 nodes were generated for the simulations to determine which of the neighborhood structures gave rise to the best boundary approximation from the WSN. For each of the hundred deployments, each of the neighborhood structures was applied.

\subsection{Experiment 1: Polygonal Region}

In a binary field, the WSN was used to monitor a simple polygonal region (Fig. 5). In order to determine the WSN's boundary approximation, a message is passed along the boundary of the region (usually the nodes just inside the boundary). 
The message collects a list of boundary coordinates as it moves from node to node along the region boundary. The message is passed anticlockwise along the region boundary - this was an arbitrary choice. The ordered list of coordinates that results from this message is the WSN's polygonal approximation of the actual region boundary.

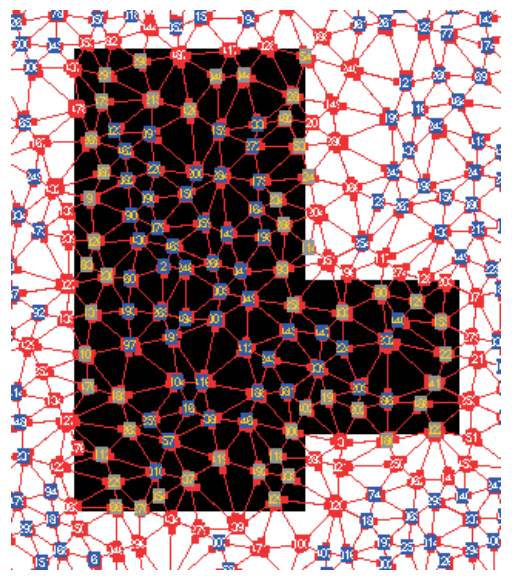

Fig. 5. Example of LGT-networked WSN monitoring a polygonal region

Figure 6 can be used to demonstrate how the WSN builds its polygonal approximation of the region boundary. The nodes maintain their neighbor tables sorted by cyclic ordering around the node. For example, $B$ 's anticlockwise neighbor list may be $(I, A, C, H)$. Let $B$ be the initiator of the boundary approximation task. From the ordered list of its neighbors, $H$ is the first neighbor of $B$ that is outside the region (since it directly follows a neighbor that is inside the region). $B$ starts the boundary polygon with the midpoint of $B H$. It then adds midpoint of $B I$ to the polygon. Since the next neighbor in the list, $A$, is inside the region, the list of coordinates is passed to $A$. $A$ adds the midpoints of $A J$, $A K, A E$ and $A D$ to the list, then passes it on to $C$. When the list of coordinates returns to $B$, it receives the complete estimate of the boundary polygon.

Results Figure 7 (one of 100 simulations) gives a qualitative view of the WSN's approximation of the monitored region's boundary using the different neighborhood structures. Table 1 gives the average $L^{2}$ error norm for the 100 simulations for each neighborhood structure. The results show that RNG is the best at estimating a region boundary, while LGT is the worst.

T-tests were performed to determine whether the differences between the various neighborhood structures were statistically significant at the $5 \%$ level. The tests revealed that they were significantly different, even LDT and LGT, which were very close in results with a 0.001 difference in $L^{2}$ error norm. 


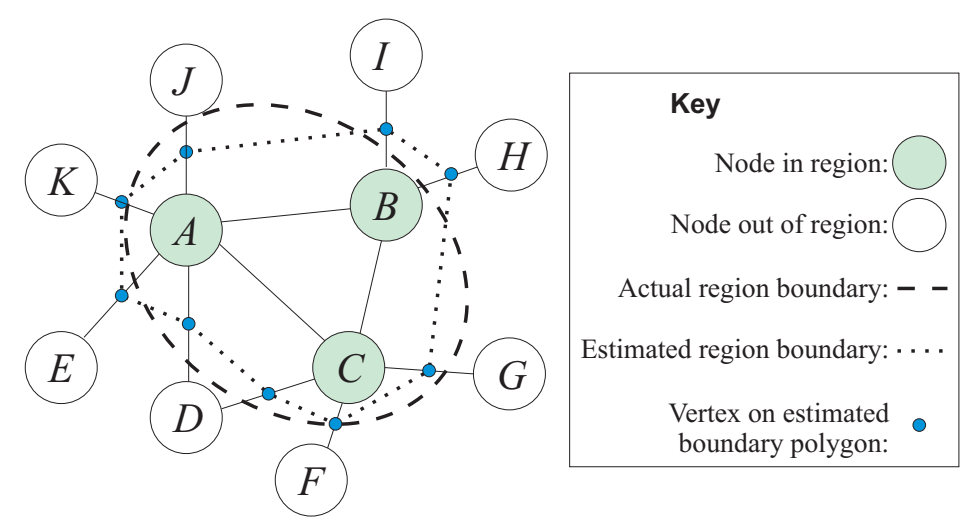

Fig. 6. A simple example of a WSN's polygonal estimate of a region boundary

Table 1. Average $L^{2}$ error norms for the polygonal region

\begin{tabular}{|c|c|}
\hline Neighborhood Structure & Average $L^{2}$ error norm \\
\hline LGT & 0.106 \\
LDT & 0.105 \\
GG & 0.0971 \\
RNG & 0.0919 \\
\hline
\end{tabular}

\begin{abstract}
Absence of UDG from Results Observe that the results do not include the UDG. This is due to the nature of the message transmission scheme in Sect. 5.1 (tracing around a boundary), which is a form of face routing since messages are sent along edges according to their cyclic order. This has the pitfalls of geographic face routing, and so non-planar graphs cause the message to loop forever. An example is shown in Fig. 8. A message arrives at $D(1)$, is passed to $B(2)$, then to $A(3), C(4)$ and back to $B(5)$, at which point it continues along path 3-4-5. Observe that removing either the edge $D B$ or $C A$ (making the graph planar) can solve the problem. The UDG is intrinsically non-planar, and so is unsuitable to the distributed boundary approximation algorithm above.
\end{abstract}

Discussion It was expected that the neighborhood structures with higher edge density (such as LDT and LGT) would perform better because the higher number of edges would allow the network to trace around the boundary more accurately. However, the results showed that RNG performed the best.

A reason for this unexpected result is that RNG is the sparsest of the four neighborhood structures. It therefore has fewer edges that intersect the region's boundary, and consequently the WSN's estimate of the region boundary has fewer vertices. This results in fewer and longer edges in the approximation polygon. This seemed a good explanation for why RNG was best at monitoring the simulated region of few vertices and long linear edges 5 . 


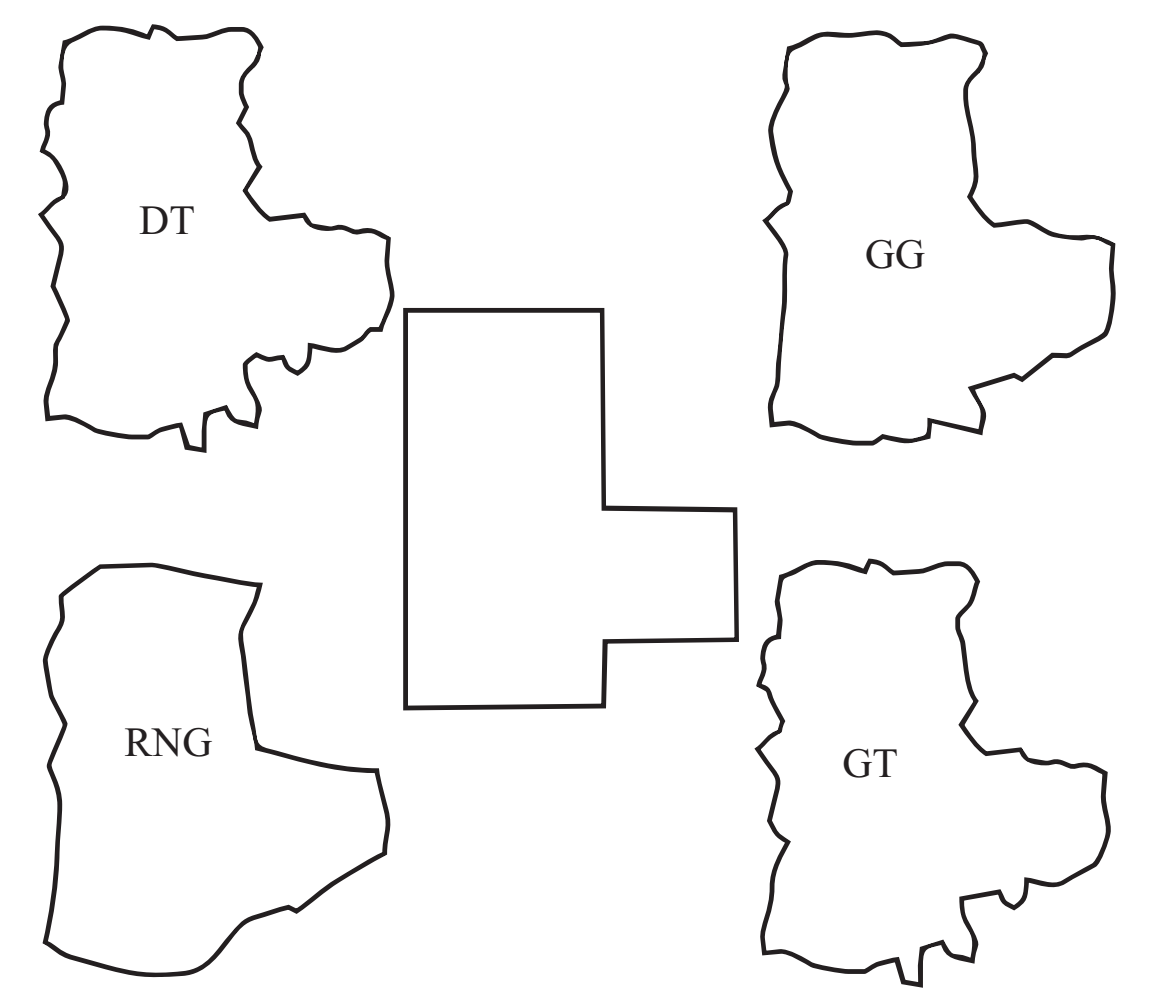

Fig. 7. Approximations of the polygonal region boundary using different neighborhood structures

\subsection{Experiment 2: Circular Region}

The results of Experiment 1 suggested that shapes with linear edges were best monitored by a WSN with RNG. Therefore, experiment was repeated with a region without straight lines - the circular region in Fig. 9.

Results Figure 10 is a sample of the shape of the boundary approximations due the the four neighborhood structures.

The $L^{2}$ error norm, averaged over 100 simulation runs, showed that RNG has the best results again (Table 2). The results were statistically significant at the $5 \%$ level, and they were also correlated - whenever one neighborhood structure work well, the others work well too (Pearson correlations of between 0.845 and 0.942). The exception was RNG, which was comparatively uncorrelated (Pearson correlations of between 0.578 and 0.674 ).

Discussion Contrary to our expectations, RNG performed better in both circular and polygonal regions. An explanation for this is that RNG is sparser than 


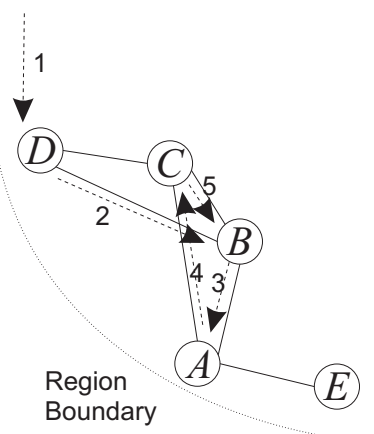

Fig. 8. Non-planar neighborhood causes an infinite loop

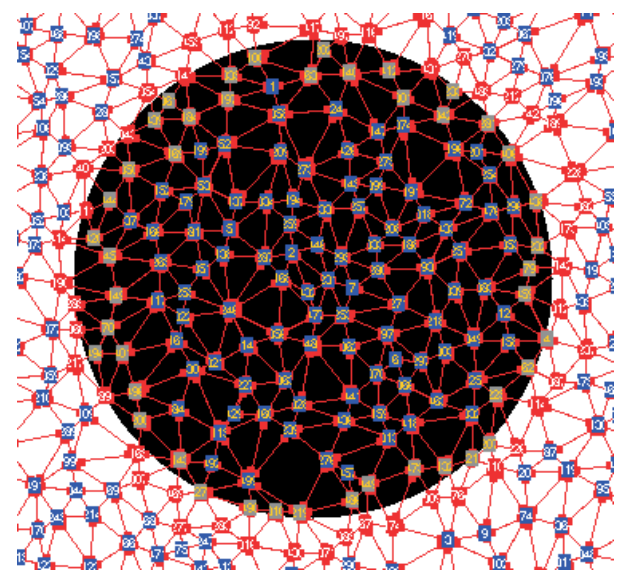

Fig. 9. Example of GT-networked WSN monitoring a circular region

the other structures in terms of edge density. Its edges are therefore shorter than those of GG and DT, of which it is a subgraph. Whenever one of the RNG edges intersect the region boundary, the likelihood is that any point on the edge is closer to the actual boundary location than any point on the longer edges of LDT. Due to this low average edge length of RNG, its boundary points were on average the most accurate.

The results for the circular region were better than the polygonal region due to the sharp corners of the polygonal region. The WSN is not always able to estimate correctly these sharp turns of the polygon boundary.

\section{Detection of Topological Events}

It has been seen that the low edge density, and consequently the low average edge length, of RNG makes it suited to locating the boundary. The following 


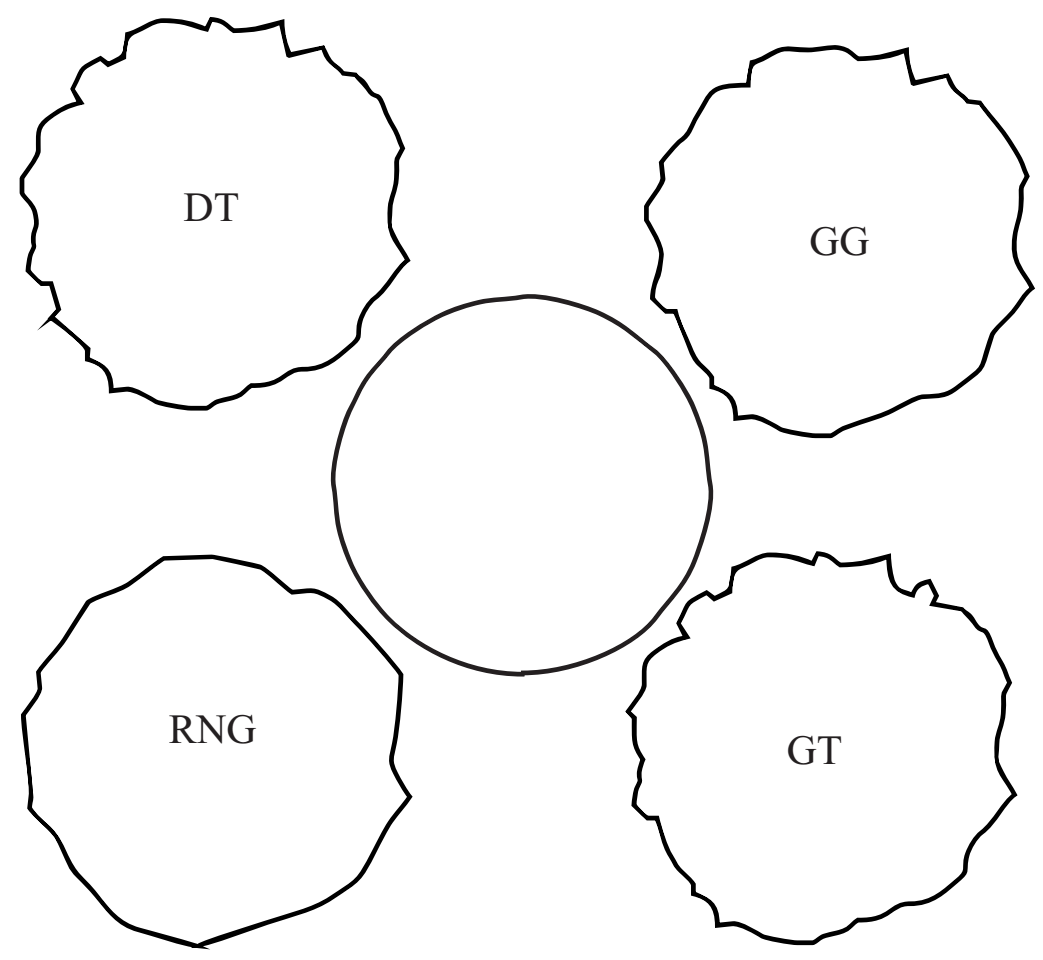

Fig. 10. Approximations of the circular region boundary using different neighborhood structures

experiments determine how the neighborhood structures perform in detecting topological events.

\subsection{Experiment 3: Merge}

In these experiments, a small square region component moves until it merges with the polygonal region component (Fig. 11). For each neighborhood structure, we ran the simulation with ten different random deployments of nodes. For each simulation run, we counted how many merges were detected.

Results WSNs under LDT, GG and LGT detected exactly one merge corresponding to the merge event in Fig. 11. RNG, however, detected 12 additional merges (Table 3).

Discussion Figure 12 demonstrates how a WSN may incorrectly identify a merge. Figure 12(a) shows a region expanding. Figure 12(b) shows the WSN's belief of what is occurring. Since $A$ and $C$ are disconnected, their detections of the region are treated as separate. So $C$ believes that a new region component 
Table 2. Average $L^{2}$ error norms for the circular region

\begin{tabular}{|c|c|}
\hline Neighborhood Structure & Average $L^{2}$ error norm \\
\hline LGT & 0.0689 \\
LDT & 0.0683 \\
GG & 0.0618 \\
RNG & 0.0508 \\
\hline
\end{tabular}

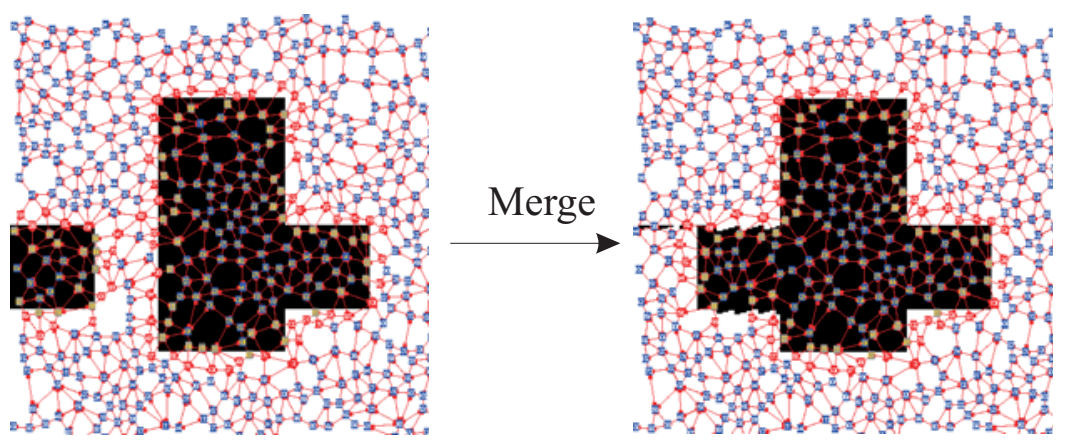

Fig. 11. Simulation of merge

has appeared. When the region expands to include $B$, it sees two distinct region components, one at $A$ and the other at $C$, come together, so it identifies it as a merge.

If the neighborhood structure had been denser, with an edge between $A$ and $C, C$ would have been able to correctly identify the change as an expansion due to having a neighbor that was in the region previously. Thus, the sparsity of the network graph causes false positives in detecting a merge. This is why RNG gives 12 false merge detections in addition to the 10 correct merges.

\subsection{Experiment 4: Split}

After the merge, the part of the region to the right splits from the region (Fig. 13). Ten different random placements of nodes were generated and then used to

Table 3. Number of merges detected with each neighborhood structure in 10 simulation runs

\begin{tabular}{|c|c|}
\hline Neighborhood Structure & Number of merges detected \\
\hline LGT & 10 \\
LDT & 10 \\
GG & 10 \\
RNG & 22 \\
\hline
\end{tabular}




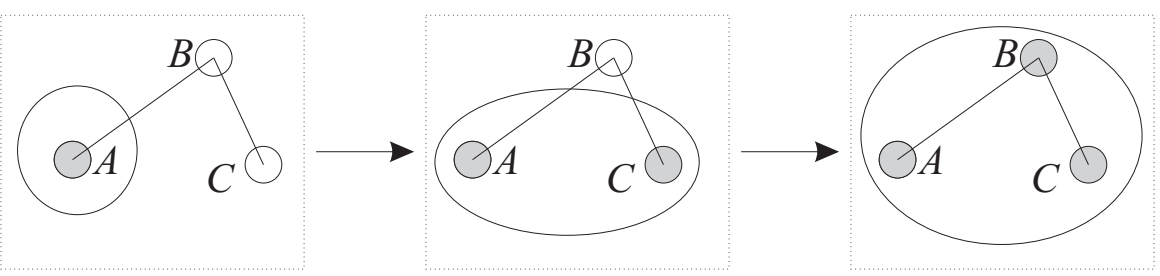

(a) Actual event: region expanding

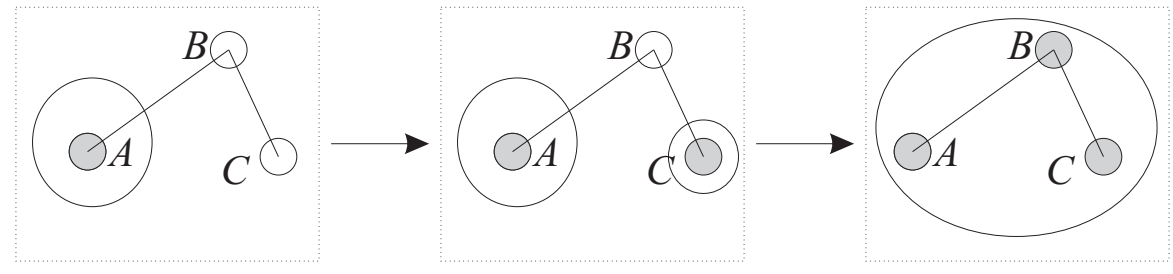

(b) WSN observes appearance and merge

Fig. 12. False detection of merge

run simulations for each neighborhood structure. The number of splits detected by the WSN was recorded.

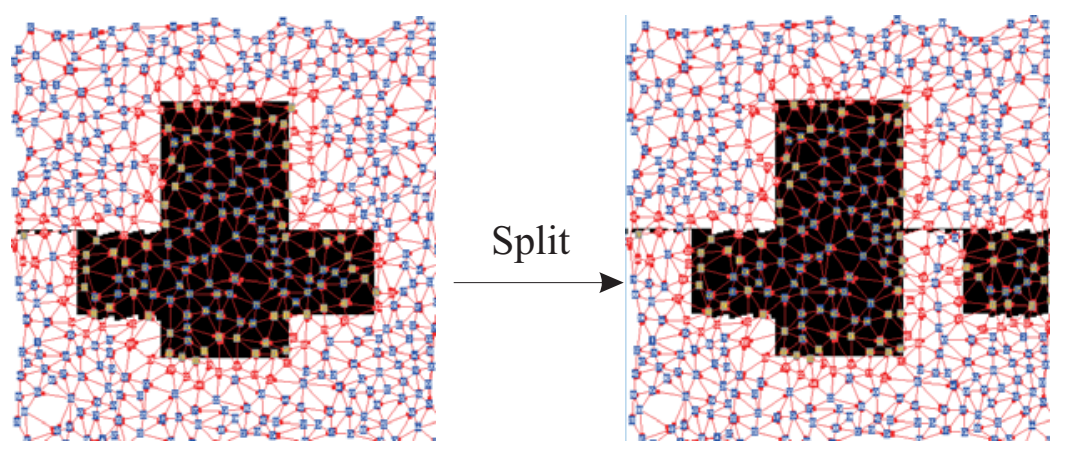

Fig. 13. Simulation scenario for detecting splits

Results Table 4 shows how many splits were missed by the WSN when the four neighborhood structures were applied. LDT and LGT did not miss any splits. GG missed 6 splits and RNG did not manage to detect a single split.

Discussion Section 6.2 explains why a node needs to have at least four neighbors in order to detect a split. Figure 2 shows that with GG, nodes do not always 
Table 4. Number of splits detected with each neighborhood structure in 10 simulation runs

\begin{tabular}{|c|c|}
\hline Neighborhood Structure & Number of splits detected \\
\hline LGT & 10 \\
LDT & 10 \\
GG & 4 \\
RNG & 0 \\
\hline
\end{tabular}

have four neighbors. With RNG, there are even more nodes with the same problem. Therefore, although GG did sometimes successfully detect a split, RNG did not detect a single one. For a node at the pinch point where a split occurred, there were never four neighbors when RNG was used.

A Further Reason for the Exclusion of UDG Section 5.1 gives routing difficulties as the reason for the exclusion of UDG. A different reason for the inapplicability of UDG in Experiments 3 and 4 is that non-planar graphs result in inconsistencies in the WSN's knowledge if we use the qualitative approach in Sect. 1. In Fig. 14, if $A$ and $C$ have an edge between them, they assume that the region is continuous between them. If there is an edge between $B$ and $D$, the region is discontinuous between $A$ and $C$ - it may be said that there is a hole in the region between $B$ and $D$. If there are edges between all the nodes, from $A$ 's and $B$ 's points of view, the region is continuous, while to $B$ and $D$, the region is discontinuous. This inconsistency in the WSN's beliefs causes problems when the nodes need to collaborate in various tasks. For these reasons, when we attempted to apply our algorithm to a UDG-networked graph, the network reported many false events and resulted in an incorrect network state. Therefore, we do not use UDG to detect topological events.

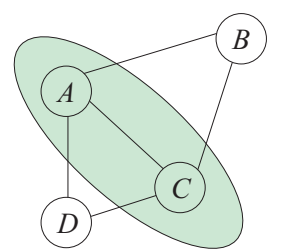

Region between $A$ and $C$

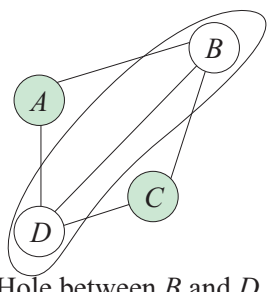

Hole between $B$ and $D$

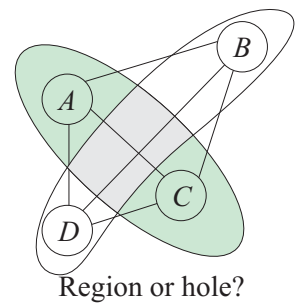

Region or hole?

Fig. 14. Inconsistency due to non-planar graph 


\section{Summary}

- It is not possible to use UDG in a WSN where routing and event detection is a concern, at least with the algorithms used in this paper.

- RNG gives the best quantitative results due to short average edge lengths, but it performs very poorly in detecting topological events. However, RNG performs poorly at qualitatively detecting topological events, to the point that it cannot be recommended for such tasks.

- LDT and LGT give the best qualitative results for event detection due to having sufficient neighbors to distinguish complex topological and nontopological events.

\section{Conclusion}

Processing of data in a WSN to generate information is an important tool for information management for various reasons, such as to improve scalability and for in-network usage of higher level information. Neighborhood structures are important for the in-network processing of spatial information. The results demonstrate that the properties of the neighborhood structure can have a significant influence on the qualitative and quantitative observations of the WSN.

When network edges are used to give spatial context to sensor readings (such as build regions), the length of the edges affects the quantitative estimates made by the WSN. Longer edges give the WSN more opportunities to make mistakes about assumptions any estimated made along the edge. Even so, other than UDG, none of the neighborhood structures failed at quantitative tasks, so there is some flexibility in choosing an appropriate structure for a WSN when other factors are taken into account.

However, qualitative comparisons rely heavily on ordering of information. Therefore, the lack of sufficient information to be ordered can give rise to false results. For this reason, the RNG fails at the split detection task, while GG performs poorly. RNG also gives false positives in detecting merges.

LDT and LGT in particular perform alike in the qualitative tasks and LDT is slightly better at quantitative tasks. If there are no other considerations (such as computational complexity), LDT should always be chosen over LGT. As for the others, researchers and engineers need to choose a structure that best fits their applications.

In our current research, we are developing the algorithm (part of which was presented in Sect. 5) to detect qualitative spatial events. From the results of this paper, we will be using LDT for our future investigations.

\section{References}

1. Mainwaring, A.M., Culler, D.E., Polastre, J., Szewczyk, R., Anderson, J.: Wireless Sensor Networks for Habitat Monitoring. In: Proc First ACM International Workshop on Wireless Sensor Networks and Applications. (2002) 88-97 
2. Hamilton, M.P., Graham, E., Rundel, P.W., Allen, M.F., Kaiser, W., Hansen, M.H., Estrin, D.L.: New Approaches in Embedded Networked Sensing for Terrestrial Ecological Observatories. Environmental Engineering Science 24(2) (2007)

3. Hart, J.K., Martinez, K.: Environmental Sensor Networks: A Revolution in the Earth System Science? Earth-Science Reviews 78 (2006) 177-191

4. Duckham, M., Nittel, S., Worboys, M.F.: Monitoring Dynamic Spatial Fields Using Responsive Geosensor Networks. In: GIS'05: Proc 13th Annual ACM International Workshop on Geographic Information Systems, New York, NY, USA, ACM Press (2005) 51-60

5. Chintalapudi, K., Govindan, R.: Localized Edge Detection in Sensor Fields. Ad Hoc Networks 1(2-3) (2003) 273-291

6. Jin, G., Nittel, S.: NED: An Efficient Noise-Tolerant Event and Event Boundary Detection Algorithm in Wireless Sensor Networks. In: MDM '06: Proceedings of the 7th International Conference on Mobile Data Management (MDM'06), Washington, DC, USA, IEEE Computer Society (2006) 153

7. de Berg, M., van Kreveld, M., Overmars, M., Schwarzkopf, O.: Computational Geometry: Algorithms and Applications. Springer-Verlag (1997)

8. Barnett, V.: Environmental Statistics: Methods and Applications. Wiley (2004)

9. Karp, B., Kung, H.T.: GPSR: Greedy Perimeter Stateless Routing for Wireless Networks. In: MobiCom '00: Proceedings of the 6th Annual International Conference on Mobile Computing and Networking, New York, NY, USA, ACM Press (2000) 243-254

10. Kim, Y.J., Govindan, R., Karp, B., Shenker, S.: On the Pitfalls of Geographic Face Routing. In: Proceedings of the Third ACM/SIGMOBILE International Workshop on Foundations of Mobile Computing, New York, NY, USA, ACM Press (September 2005) 34-43

11. Preparata, F.P., Shamos, M.I.: Computational Geometry: An Introduction. Springer-Verlag New York, Inc., New York, NY, USA (1985)

12. Zhao, F., Guibas, L.: Wireless Sensor Networks: An Information Processing Approach. Elsevier/Morgan-Kaufmann, Amsterdam (2004)

13. Vaidyanathan Ramadurai, M.L.S.: Localization in Wireless Sensor Networks: A Probabilistic Approach. In Zhuang, W., Yeh, C.H., Droegehorn, O., Toh, C.T., Arabnia, H.R., eds.: Proceedings of the International Conference on Wireless Networks, Las Vegas, Nevada, USA, CSREA Press (June 2003) 275-281

14. Li, X.Y., Calinescu, G., Wan, P.J., Wang, Y.: Localized Delaunay Triangulation with Application in Ad Hoc Wireless Networks. IEEE Transactions on Parallel and Distributed Systems 14(10) (2003) 1035-1047

15. Funke, S., Milosavljevic, N.: Infrastructure-Establishment from Scratch in Wireless Sensor Networks. In: Distributed computing in sensor systems : First IEEE International Conference, DCOSS 2005. Volume 3560 of Lecture Notes in Computer Science., Marina Del Rey, USA (2005) 354-367

16. Jiang, J., Worboys, M.F.: Event-Based Topology for Dynamic Planar Areal Object. International Journal of Geographical Information Science (2008) Under review. 